\title{
BMJ Open Pretreatment with transcutaneous electrical acupoint stimulation to prevent postoperative ileus in patients undergoing laparoscopic colon surgery: study protocol for a randomised controlled trial
}

Jian Wang, ${ }^{1}$ Dongli Li, ${ }^{2}$ Wei Tang, ${ }^{1}$ Jun Guo, ${ }^{1}$ Wenting Chen, ${ }^{1}$ Yue Yong, ${ }^{3}$ Wei Song, ${ }^{1}$ Guijie Yu, ${ }^{1}$ Rui Feng, ${ }^{1}$ Lan Yuan, ${ }^{1}$ Guoqiang Fu, ${ }^{1}$ Jiangang Song, ${ }^{1}$ Lihua Fan (1) ${ }^{2}$

To cite: Wang J, Li D, Tang W, et al. Pretreatment with transcutaneous electrical acupoint stimulation to prevent postoperative ileus in patients undergoing laparoscopic colon surgery: study protocol for a randomised controlled trial. BMJ Open 2020;10:e030694. doi:10.1136/ bmjopen-2019-030694

- Prepublication history and additional material for this paper are available online. To view these files, please visit the journal online (http://dx.doi. org/10.1136/bmjopen-2019030694).

JW and DL contributed equally.

Received 13 April 2019

Revised 09 May 2020

Accepted 10 June 2020

Check for updates

(c) Author(s) (or their employer(s)) 2020. Re-use permitted under CC BY-NC. No commercial re-use. See rights and permissions. Published by BMJ.

For numbered affiliations see end of article.

Correspondence to

Dr Lihua Fan;

fanlihua_Is@126.com

\section{ABSTRACT}

Introduction Postoperative ileus (POI), a common complication after surgery, severely affects postoperative recovery. It is unclear whether pretreatment with transcutaneous electrical acupoint stimulation (TEAS) can improve recovery from $\mathrm{POI}$. This trial will evaluate the effects of pretreatment with TEAS on POI.

Methods and analysis This will be a prospective, randomised controlled trial. American Society of Anesthesiologists (ASA) physical status classification I-III level patients, aged 18-75 years and scheduled for laparoscopic colon surgery, will be included in the study. It is planned that 146 subjects will be randomised to the TEAS and sham TEAS (STEAS) groups. The groups will undergo two sessions of TEAS/STEAS daily for 3 days before surgery, with a final TEAS/STEAS treatment $30 \mathrm{~min}$ before anaesthesia. The primary endpoint of the study will be time to first defaecation. Secondary endpoints will include time to first flatus, time to tolerance of oral diet, Gl-2 (composite outcome of time to first defaecation and time to tolerance of oral diet), time to independent walking, length of hospital stay, postoperative pain Visual Analogue Scale score on the first 3 days after surgery, analgesic requirements, complications and plasma concentrations of interferon- $\beta$ (IFN- $\beta$ ), IFN- $\gamma$, interleukin- 6 (IL-6) and IL-1 $\beta$. Multiple linear regression will be used to identify independent predictors of outcome measures.

Ethics and dissemination This study has been approved by the Chinese Registered Clinical Trial Ethics Review Committee (No. ChiECRCT-20170084). The results of the trial will be published in an international peer-reviewed journal.

Trial registration number This study has been registered with the Chinese Clinical Trial Registry (No. ChiCTR-

INR-17013184).

Trial status The study was in the recruitment phase at the time of manuscript submission.

\section{INTRODUCTION}

Postoperative ileus (POI) is a transient dysfunction of gastrointestinal (GI)
Strengths and limitations of this study

- This study aims to evaluate whether pretreatment with transcutaneous electrical acupoint stimulation (TEAS) can prevent postoperative ileus (POI).

- TEAS is a safe, non-invasive and easily accepted adjunctive intervention.

- This study will provide deeper insights into the mechanism by which TEAS pretreatment reduces the inflammatory response.

- This is a single-centre study, which is a potential limitation.

propulsion that often occurs after abdominal surgery and may also occur after surgery at other sites. ${ }^{1}$ The main symptoms of POI include abdominal pain and distention, nausea, vomiting, difficult defaecation and intolerance to solid food. POI is usually temporary, but if prolonged, may lead to surgical incision dehiscence, intestinal anastomotic fistula, abdominal cavity infection, intestinal ischaemia, aspiration pneumonia and other serious complications. ${ }^{2-4}$ A retrospective cohort study involving nearly 500 hospitals in the USA showed that POI is a key reason for prolonged hospitalisation and increased medical costs for patients undergoing abdominal surgery. ${ }^{1}$ The USA spends more than $\$ 1.46$ billion treating POI every year. ${ }^{5}$ At present, the most common methods used to treat POI include rational perioperative use of narcotic drugs and opioids, eating as soon as possible after surgery, avoidance of nasogastric tubes after the operation, early ambulation, postoperative epidural analgesia, restriction of fluid intake, the use 
of minimally invasive surgery (such as laparoscopic), drug therapy and the use of chewing gum. Despite the numerous treatment strategies, POI remains a difficult clinical challenge that compromises the rapid recovery of postoperative patients. It is, therefore, necessary to find more effective, convenient and economical treatment methods. ${ }^{6-10}$

The main mechanism underlying POI may be activation of macrophages in the external muscular layer during the surgical procedure. ${ }^{11}$ Intestinal manipulation during surgery can activate macrophages in the outer muscle layer of the small intestine, leading to release of inflammatory factors (interleukin-6 (IL-6), IL-1 $\beta$ ) and the chemokine MIP- $1 \alpha$, together with increased expression of the adhesion molecule ICAM-1 on endothelial cells and induction of neutrophils and monocytes in the circulation into the small intestine muscle layer. These cells, and activated macrophages, can release a large amount of inducible nitric oxide synthase and prostaglandin, which inhibit the movement and contraction of the GI tract. ${ }^{12} 13$ Transport of these inflammatory mediators in the bloodstream causes activation of macrophages in the distal GI tract, leading to POI over the entire intestinal tract. ${ }^{14}$ It has been confirmed by a large number of animal experiments that reducing the inflammatory response is an effective way to treat POI. ${ }^{15-17}$

There is a long history in traditional Chinese medicine (TCM) of using acupuncture to treat functional GI diseases and, in recent years, there has been significant global interest in the beneficial effects of acupuncture on POI. The positive effect of electroacupuncture (EA) on POI has been clearly demonstrated. Ng et al used EA to treat POI in patients undergoing laparoscopic colon surgery. ${ }^{18}$ Defaecation time and length of hospital stay were significantly shortened in patients who received EA compared with those who did not receive the treatment. In patients undergoing hepatic resection, You et al found a significant reduction in the incidence of $\mathrm{POI}$ in patients treated with a combination of acupuncture and Chinese herbal medicine. The length of hospitalisation was also significantly shortened in the treated group $(14.0 \pm 4.9$ days vs $16.5 \pm 6.8$ days, $\mathrm{p}=0.014){ }^{19}$

In the previous studies, we proved that pretreatment with acupuncture could reduce excessive activation of the innate immune system and inhibit the inflammatory response. This effect may be achieved by activation of the vagal nervous system. ${ }^{20} 21$ Other studies have shown that transcutaneous electrical acupoint stimulation (TEAS) and EA have similar effects in the treatment of pain and alleviating the inflammatory response. ${ }^{22} 23$

TCM holds that the best treatment for disease is prevention. Based on all of the above studies, we hypothesise that the use of TEAS as a preoperative treatment may reduce the incidence of POI. There have, so far, not been any studies that address this question.

We have, therefore, designed a randomised controlled trial to investigate whether pretreatment with TEAS can reduce the incidence of POI in patients undergoing laparoscopic colon resection. The study is also designed to verify that the anti-inflammatory effect is associated with the immunomodulatory function of TEAS.

\section{METHODS AND ANALYSIS Study objective}

The primary objective is to assess the effect of TEAS on clinical recovery of bowel function after laparoscopic colon surgery. The secondary objective is to verify that suppression of overactivation of the innate immune system and reduction of the inflammatory response are the mechanisms underlying the ability of pretreatment of percutaneous acupuncture to prevent POI.

\section{Study location}

A prospective, single-centre, double-blinded, randomised controlled trial will be conducted at Shuguang Hospital, which is affiliated to the Shanghai University of Traditional Chinese Medicine, China.

\section{Study population}

Participants will be recruited according to the inclusion and exclusion criteria.

Inclusion criteria:

1. Male and female patients aged $18-75$ years.

2. Patients undergoing elective laparoscopic colonic surgery and upper rectal resection (such as left collect, right colectomy and anterior resection of the upper part of the rectum and lower part of the sigmoid).

3. Body mass index $18-31 \mathrm{~kg} / \mathrm{m}^{2}$.

4. ASA classification I-III.

5. Patients provide signed informed consent (the consent form can be viewed in online supplementary appendix 1).

Exclusion criteria:

1. Middle and lower rectal resection, total/proctocolectomy or the need for complex endoscopic surgery.

2. Need for abdominal wall fistula, GI fistula, fistula surgery or stoma creation.

3. History of abdominal/pelvic operations or complications.

4. Patients receiving epidural anaesthesia or epidural analgesia.

5. Patients with skin infections, surgical incision or scar at the point of application of acupuncture.

6. Patients have a history of limb surgery, spinal surgery or nerve injury.

7. Patients who participated in other clinical trials, or received other acupuncture therapy, in the previous 4 weeks.

8. Patients with cardiac pacemakers.

9. Patients have one of the following conditions before surgery: chronic pain, drug addiction or alcohol dependence.

10. Patients with preoperative combination of severe central nervous system disease and severe mental illness. 


\section{ENDPOINTS}

\section{Primary endpoint}

First defaecation time (h), that is, time to first anal defaecation after laparoscopic surgery.

\section{Secondary endpoints}

Time to first flatus (h), time to tolerance of solid oral diet (h), GI-2 (composite outcome of time to first defaecation and time to tolerance of oral diet), time to walk independently $(\mathrm{h})$, length of hospital stay, defined as the number of days from operation to discharge (d). Criteria for hospital discharge include stability of vital signs with no fever, achievement of flatus or defaecation, ability to tolerate solid food without vomiting, control of postoperative pain, absence of other postoperative complications and ability to function at home independently or with home care provided. Pain will be assessed using the Visual Analogue Scale (VAS) on postoperative days 1, 2 and 3 (scale of 0 to 10, where 0 represents complete absence of pain and 10 represents the worst pain intensity). Postoperative requirements for analgesia will also be assessed. Inflammatory mediators (interferon- $\beta$ (IFN- $\beta$ ), IFN- $\gamma$, interleukin-6 (IL-6) and IL-1 $\beta$ ) in blood will be measured before TEAS/STEAS intervention and on days 1, 3 and 5 after the operation. Postoperative complications will be recorded using the Clavien-Dindo classification for complication assessment. ${ }^{24}$ The follow-up period will be at least 6 months.

We add GI-2 as a secondary outcome to the original protocol after recruitment of the study had already begun. GI-2 is a time indicator, which will be calculated from two existing outcomes (time to first defaecation and time to tolerance of oral diet). There will be no harm to subjects, no additional cost and no more work.

\section{Randomisation and blinding}

Patients will be randomised to receive either TEAS or STEAS by stratified randomisation according to sex, in a 1:1 ratio (figure 1). Using a computer-generated random sequence, a sealed envelope will be opened to determine to which group the patient has been assigned. The acupuncturist will be aware of the treatment group. Patients, as well as the outcome investigator (nurse anaesthetist), will be blinded to the treatment allocation.

\section{Current sample size justification}

According to Wang Jian and Song Jiangang's preliminary study of TEAS pretreatment for prevention of POI in patients undergoing laparoscopic colon surgery in Shuguang Hospital, the mean time to first defaecation following laparoscopic colon surgery was $62 \pm 19$ hours $(\mathrm{M} \pm \mathrm{SD})$. Working on the assumption that a clinically meaningful difference in mean time to first defaecation between the TEAS and STEAS groups is 1 day or 24 hours, 66 patients would be needed in each group to reach a power of $80 \%$ and a $5 \%$ type I error rate. If the dropout rate is $10 \%$, a total sample size of 146 patients for the two groups is needed for this study.

\section{Statistical analysis}

Data for continuous variables (ie, first defaecation time, first passage of flatus, time to tolerance of oral diet, time to walking independently, length of hospital stay) will be reported using the mean and $\mathrm{SD}(\mathrm{M} \pm \mathrm{SD})$ for normally distributed data or median (range) for skewed data. Data for categorical variables will be expressed as a number (percentage). Intergroup differences will be assessed using the Student's t-test or Mann-Whitney U test. Intergroup differences in inflammatory mediators (at time points of pre-TEAS/STEAS treatment, and on

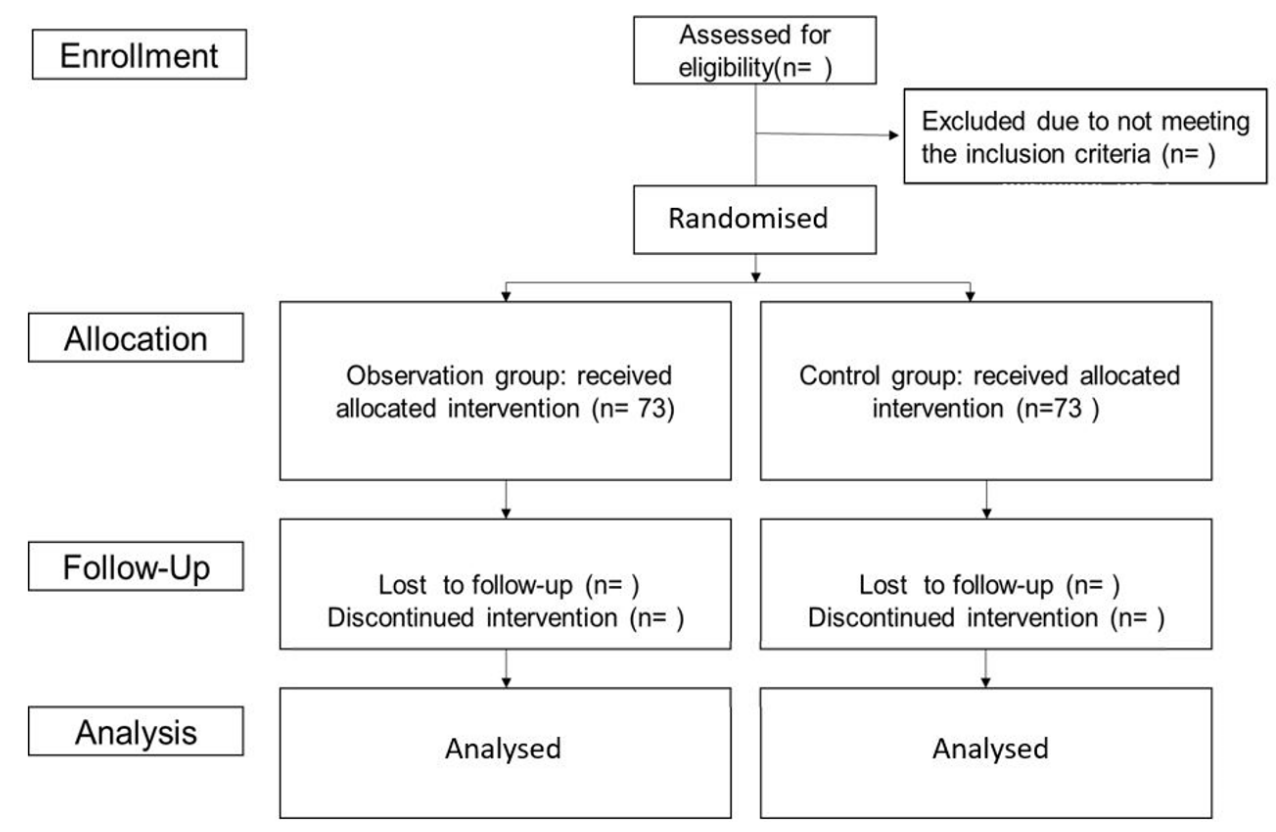

Figure 1 Flowchart of the study protocol. 


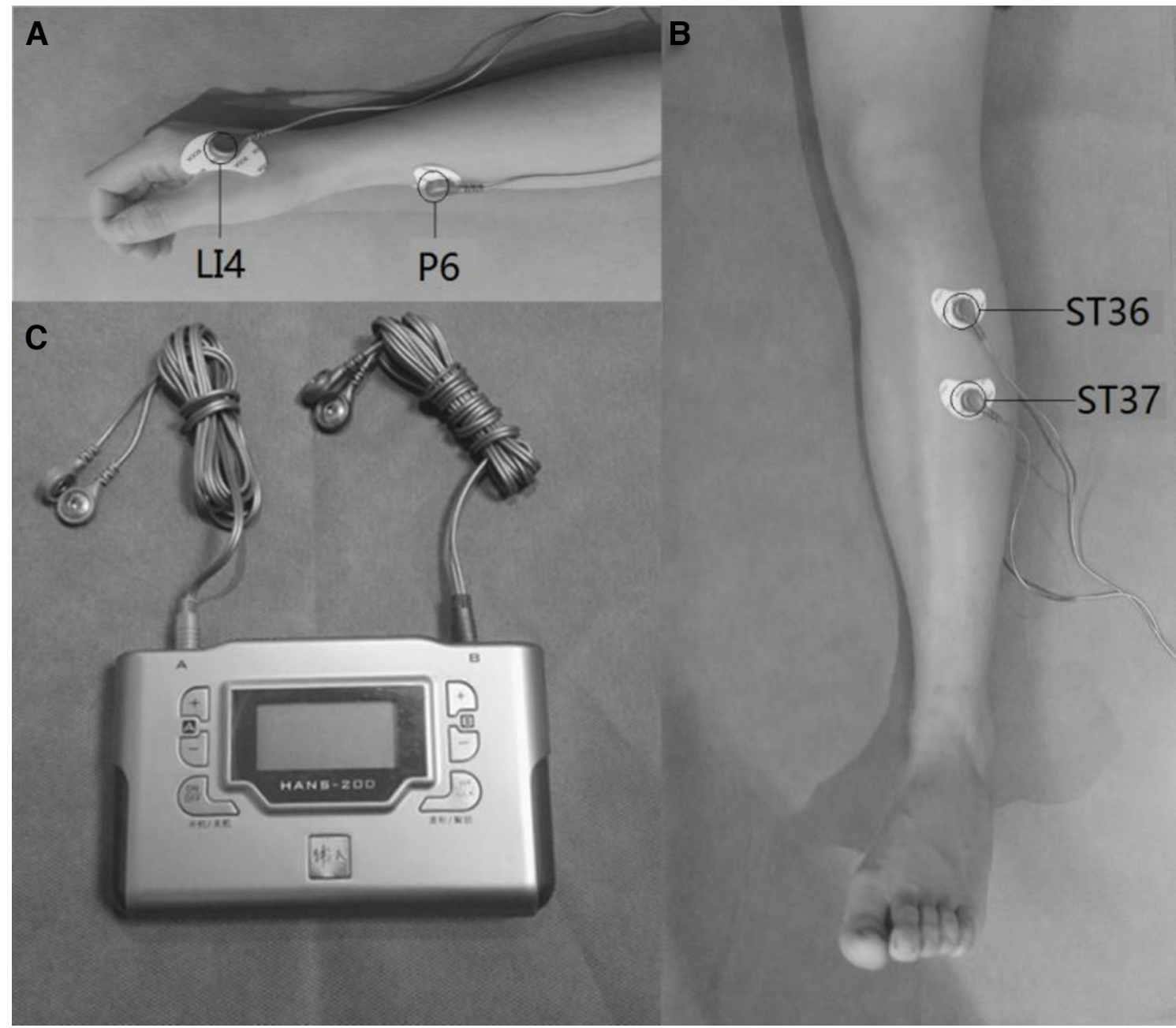

Figure 2 Acupoints selected in this trial: (A) Hegu (IL-4) and Neiguan (P-6); (B) Zusanli (ST-36) and Shangjuxu (ST-37); (C) Han's acupoint nerve stimulator.

postoperative days 1, 3 and 5) were assessed by two-way repeated measures analysis of variance with Bonferroni post hoc test. The significance level will be set at $5 \%$. All data will be analysed using SPSS V.17.0 software or other appropriate statistical software packages.

\section{Pretreatment}

Patients randomised to the TEAS and STEAS groups will undergo two treatment sessions daily for three consecutive days before surgery. The patients will then be treated for a final time $30 \mathrm{~min}$ before anaesthesia.

For patients in the TEAS group, the Zusanli (ST-36), Shangjuxu (ST-37), Hegu (LI-4) and Neiguan (P-6) acupoints will be identified before electrical stimulation with surface electrodes (figure 2). Selection of these acupoints is based on a consensus between the acupuncturists carrying out the study. The acupuncturist will stimulate these acupoints using a Han's acupoint nerve stimulator (HANS200A, Nanjing Jisheng Medical Technology, Nanjing, China) at a frequency of $100 \mathrm{~Hz}$. The intensity will be adjusted for each individual to maintain a slight twitching of the regional muscle and achieve De-Qi sensations, such as soreness, numbness, distention and heaviness. The STEAS group will receive a strong, but comfortable current for $30 \mathrm{~s}$, and the current will then gradually vanish over the next $15 \mathrm{~s}^{25}$ The participants of both groups will be told that they are receiving current stimulation. Each session of acupoints treatment will last for $30 \mathrm{~min}$. During the application of TEAS, patients will be required not to change the current settings themselves. A prompt beep at the end of TEAS will indicate the end of treatment.

All surgery will be carried out under general anaesthesia using standardised anaesthetic procedures. Patients will be fasted for 12 hours before surgery. Right upper extremity venous access will be established before the patients entering the operating theatre. Ringer's lactate solution $(8 \mathrm{~mL} / \mathrm{kg})$ will be administered by intravenous infusion for compensatory expansion before induction of anaesthesia. Patients will then receive midazolam $(0.04 \mathrm{mg} / \mathrm{kg})$, fentanyl $(3 \mu \mathrm{g} / \mathrm{kg})$, vecuronium bromide $(0.1 \mathrm{mg} / \mathrm{kg})$ and propofol $(1.5-2.0 \mathrm{mg} / \mathrm{kg})$ intravenously for induction of anaesthesia. Anaesthesia will be maintained using a CP-600 anaesthesia delivery system (Slgo Medical Technology, Beijing, China). The dose 
of propofol will be adjusted to maintain the bispectral index in the range of 40-60. After surgery, all patients will remain in the post-anaesthesia care unit and then return to the ward for recovery until discharge.

The perioperative management of all patients will be standardised. Early ambulation will be encouraged and oral feeding will be resumed as early as possible. All patients will be followed up for at least 6 months after discharge from the hospital.

\section{Adverse events}

All adverse reactions will be closely monitored through spontaneous reports by patients or direct observation by clinicians, or by asking the patients about adverse events using open questions. All adverse reactions will be recorded and appropriate treatment will be provided if necessary. Serious adverse events will be reported to the ethics committee.

\section{Data collection and management}

Demographic variables and clinical data will be collected from all patients. During the study, blood pressure, heart rate and oxygen saturation will also be monitored. Any adverse events will be recorded. Data will be collected throughout the study and will be securely managed under conditions of confidentiality. Data collection will be performed by a nurse anaesthetist. The participants will be referred to by their participant number rather than by their name throughout the study, unless otherwise specified. All relevant documents and files will be archived for 5 years. The data will be accessible only by investigators who sign the confidential disclosure agreement and by institutional or governmental auditors during the study. Data without patient identifiers will be publicly accessible after the study. Data collection and management will be monitored by the Institutional Ethics Committee for Clinical Research of Shuguang Hospital.

\section{Patient and public involvement}

This study is currently in the recruitment phase. Patients and/or the public were not involved in study design or conduct of the study. The participants will be able to access the study results through social media.

\section{DISCUSSION}

POI continues to represent an important cause of morbidity after colon surgery. The prevention of POI is thus of great importance in reducing perioperative complications and reducing hospitalisation costs. Although it has been shown that EA can shorten the duration of POI, ${ }^{18}$ the effectiveness of TEAS, which is a similar technique, in preventing POI has not been investigated. It is, therefore, important to assess the effectiveness of TEAS in preventing POI through a clinical study.

This study has several strengths. First, the intervention strategy of the protocol will be pretreatment with TEAS. Previous studies have shown that pretreatment has a prophylactic effect. For example, pretreatment with TEAS has been shown to improve pain treatment ${ }^{2627}$ and to improve resuscitation after anaesthesia, with reduction of postoperative nausea and vomiting. ${ }^{28}$ It is, however, unclear whether preoperative TEAS can prevent POI. Studies suggest that early preoperative intervention may be more beneficial in regulating physiological functions and preventing POI. ${ }^{29}$ In an extension to these findings, the present study will help to determine whether TEAS pretreatment could improvement POI.

Second, the effectiveness of TEAS will be evaluated by assessing clinical function and by serological examination. In this randomised controlled trial of patients undergoing laparoscopic colorectal surgery, our aim is to assess the effects of preoperative TEAS on POI using relevant clinical parameters associated with bowel function. These include time to first defaecation, time to first flatus, time to tolerance of oral diet and GI-2. Importantly, we will also measure serum concentrations of inflammatory mediators associated with POI, such as IFN- $\beta$, IFN- $\gamma$, IL-6 and IL-1 $\beta$. Our findings may, thus, provide deeper insights into the mechanisms by which TEAS improves POI.

There are also limitations to this protocol. Various clinical indicators have been used in studies for the diagnosis of POI, but there is no consensus on which clinical parameter is the best for assessment of GI transit. ${ }^{930}{ }^{31}$ Two indicators that are widely used to assess bowel movement will be used in this study. Time to first defaecation will be the primary outcome and time to first flatus will be one of the secondary outcomes. There is a possibility that we may observe conflicting results (ie, significant improvement in time to flatus, but not defaecation). Because flatus can vary considerably between patients, clinical trials support the time to tolerance of oral diet and GI-2 (defined as the later of the following two events: time to first tolerance of solid food and time to first bowel movement) as supplementary secondary outcomes to measure the recovery time of GI function and these will be used in this study. ${ }^{32}{ }^{33}$ Other limitations of these indicators are that they require objective measurement of motility and are time consuming to measure. ${ }^{34}{ }^{35}$ Recently, this situation has been improved by the use of in vivo monitoring techniques to assess the function of GI movements. Innovative devices, such as Sitz markers, have been used to evaluate postoperative recovery of small bowel movement by counting the number of Sitz markers that did not pass through the ileocecal valve, but remained in the small intestine using radiography. ${ }^{36}$ The SmartPill is a swallowable device that record parameters within the GI tract. Indicators, such as $\mathrm{pH}$, temperature and intracavitary pressure, can be collected to analyse GI transit times in vivo. ${ }^{37}$ These devices acquire objective parameters to evaluate bowel movement and could save time. Research into the satisfaction of both doctors and patients with these device needs to be carried out. Furthermore, this study is a single-centre trial and, because the therapeutic effect of TEAS may be affected by ethnicity and region, it will 
be necessary to conduct multicentre and large sample studies in the future.

Notwithstanding its limitations, this study can clearly indicate the overall effects of TEAS on postoperative recovery. We hypothesise that pretreatment with TEAS could improve recovery of GI function in patients undergoing laparoscopic surgery. If this study provides positive results, it will be possible to recommend this pretreatment strategy for patients undergoing abdominal surgery. Relevant cost-effectiveness studies are also worthy of consideration.

\section{Author affiliations}

${ }^{1}$ Anesthesiology, Shuguang Hospital Affiliated to Shanghai University of Traditional Chinese Medicine, Shanghai, China

${ }^{2}$ Anesthesiology, Wenzhou Medical University, the sixth Affiliated Hospital, Lishui, China

${ }^{3}$ Research Institute of Acupuncture Anesthesia, Shuguang Hospital Affiliated to Shanghai University of Traditional Chinese Medicine, Shanghai, China

Acknowledgements We thank Dr Stanley Tao from Shanghai Ruihui Biotech for his valuable assistance in the statistical design of this study.

Contributors JW conceived the study. DL, WT, JG and GF participated in its design and coordination. WC, YY, WS and JG collected references and developed the protocol. GY and LY will perform statistical analyses. RF will follow-up with patients and record data. JW, LF and JS drafted the manuscript. All authors have read and approved the final manuscript.

Funding The present study is supported by the project of the National Natural Science Foundation of China (Nos. 81703898, 81603702, 81603700 and 81774108) and the commercial sponsorship of SINCH Pharmaceuticals Tech. Competing interests None declared.

Patient consent for publication Obtained.

Provenance and peer review Not commissioned; externally peer reviewed.

Open access This is an open access article distributed in accordance with the Creative Commons Attribution Non Commercial (CC BY-NC 4.0) license, which permits others to distribute, remix, adapt, build upon this work non-commercially, and license their derivative works on different terms, provided the original work is properly cited, appropriate credit is given, any changes made indicated, and the use is non-commercial. See: http://creativecommons.org/licenses/by-nc/4.0/.

ORCID iD

Lihua Fan http://orcid.org/0000-0001-5308-9617

\section{REFERENCES}

1 lyer S, Saunders WB, Stemkowski S. Economic burden of postoperative ileus associated with colectomy in the United States. $J$ Manag Care Pharm 2009;15:485-94.

2 Boelens PG, Heesakkers FFBM, Luyer MDP, et al. Reduction of postoperative ileus by early enteral nutrition in patients undergoing major rectal surgery: prospective, randomized, controlled trial. Ann Surg 2014;259:649-55.

3 Melis M, Fichera A, Ferguson MK. Bowel necrosis associated with early jejunal tube feeding: a complication of postoperative enteral nutrition. Arch Surg 2006;141:701-4.

4 Moghadamyeghaneh Z, Hwang GS, Hanna MH, et al. Risk factors for prolonged ileus following colon surgery. Surg Endosc 2016;30:603-9.

5 Goldstein JL, Matuszewski KA, Delaney CP, et al. Inpatient economic burden of postoperative ileus associated with abdominal surgery in the United States. P and T 2007;32:82-90.

6 Bragg D, El-Sharkawy AM, Psaltis E, et al. Postoperative ileus: recent developments in pathophysiology and management. Clin Nutr 2015;34:367-76.

7 Wolthuis AM, Bislenghi G, Fieuws S, et al. Incidence of prolonged postoperative ileus after colorectal surgery: a systematic review and meta-analysis. Colorectal Dis 2016;18:01-9.

8 Nguyen DL, Maithel S, Nguyen ET, et al. Does Alvimopan enhance return of bowel function in laparoscopic gastrointestinal surgery? A meta-analysis. Ann Gastroenterol 2015;28:475-80.
9 van Bree SHW, Nemethova A, Cailotto C, et al. New therapeutic strategies for postoperative ileus. Nat Rev Gastroenterol Hepatol 2012;9:675-83.

10 Hilton WM, Lotan Y, Parekh DJ, et al. Alvimopan for prevention of postoperative paralytic ileus in radical cystectomy patients: a costeffectiveness analysis. BJU Int 2013;111:1054-60.

11 Wehner S, Behrendt FF, Lyutenski BN, et al. Inhibition of macrophage function prevents intestinal inflammation and postoperative ileus in rodents. Gut 2007;56:176-85.

12 Wehner S, Straesser S, Vilz TO, et al. Inhibition of p38 mitogenactivated protein kinase pathway as prophylaxis of postoperative ileus in mice. Gastroenterology 2009;136:619-29.

13 Schwarz NT, Kalff JC, Türler A, et al. Prostanoid production via COX-2 as a causative mechanism of rodent postoperative ileus. Gastroenterology 2001;121:1354-71.

14 Engel DR, Koscielny A, Wehner S, et al. T helper type 1 memory cells disseminate postoperative ileus over the entire intestinal tract. Nat Med 2010;16:1407-13.

15 Adding LC, Bannenberg GL, Gustafsson LE. Basic experimental studies and clinical aspects of gadolinium salts and chelates. Cardiovasc Drug Rev 2001;19:41-56.

16 Koscielny A, Kalff JC. T-helper cell type 1 memory cells and postoperative ileus in the entire gut. Curr Opin Gastroenterol 2011;27:509-14.

17 Mikkelsen HB, Thuneberg L. Op/op mice defective in production of functional colony-stimulating factor- 1 lack macrophages in muscularis externa of the small intestine. Cell Tissue Res 1999;295:485-93.

18 Ng SSM, Leung WW, Mak TWC, et al. Electroacupuncture reduces duration of postoperative ileus after laparoscopic surgery for colorectal cancer. Gastroenterology 2013;144:307-13.

19 You X-M, Mo X-S, Ma L, et al. Randomized clinical trial comparing efficacy of Simo decoction and acupuncture or chewing gum alone on postoperative ileus in patients with hepatocellular carcinoma after hepatectomy. Medicine 2015;94:e1968.

20 Song JG, Li HH, Cao YF, et al. Electroacupuncture improves survival in rats with lethal endotoxemia via the autonomic nervous system. Anesthesiology 2012;116:406-14.

21 Zhang J, Yong Y, Li X, et al. Vagal modulation of high mobility group box-1 protein mediates electroacupuncture-induced cardioprotection in ischemia-reperfusion injury. Sci Rep 2015;5:15503.

22 Balogun JA, Biasci S, Han L. The effects of acupuncture, electroneedling and transcutaneous electrical stimulation therapies on peripheral haemodynamic functioning. Disabil Rehabil 1998;20:41-8.

23 Jiang $\mathrm{Y}$, Wang $\mathrm{H}$, Liu Z, et al. Manipulation of and sustained effects on the human brain induced by different modalities of acupuncture: an fMRI study. PLoS One 2013;8:e66815.

24 Dindo D, Demartines N, Clavien P-A. Classification of surgical complications: a new proposal with evaluation in a cohort of 6336 patients and results of a survey. Ann Surg 2004;240:205-13.

25 Rakel B, Cooper N, Adams HJ, et al. A new transient sham TENS device allows for investigator blinding while delivering a true placebo treatment. J Pain 2010;11:230-8.

26 Huang L, Pan Y, Chen S, et al. Prevention of propofol injectionrelated pain using pretreatment transcutaneous electrical acupoint stimulation. Turk J Med Sci 2017:47:1267-76.

27 Zhang Q, Gao Z, Wang H, et al. The effect of pre-treatment with transcutaneous electrical acupoint stimulation on the quality of recovery after ambulatory breast surgery: a prospective, randomised controlled trial. Anaesthesia 2014;69:832-9.

28 Zheng LH, Sun H, Wang GN, et al. Effect of transcutaneous electrical acupoint stimulation on nausea and vomiting induced by patient controlled intravenous analgesia with tramadol. Chin J Integr Med 2008:14:61-4.

29 Stakenborg N, Labeeuw E, Gomez-Pinilla PJ, et al. Preoperative administration of the 5-HT4 receptor agonist prucalopride reduces intestinal inflammation and shortens postoperative ileus via cholinergic enteric neurons. Gut 2019;68:1406-16.

30 Vather R, Trivedi S, Bissett I. Defining postoperative ileus: results of a systematic review and global survey. $J$ Gastrointest Surg 2013;17:962-72.

31 Wu Z, Boersema GSA, Dereci A, et al. Clinical endpoint, early detection, and differential diagnosis of postoperative ileus: a systematic review of the literature. Eur Surg Res 2015;54:127-38.

32 Deng G, Wong WD, Guillem J, et al. A phase II, randomized, controlled trial of acupuncture for reduction of Postcolectomy ileus. Ann Surg Oncol 2013;20:1164-9.

33 van Bree SHW, Bemelman WA, Hollmann MW, et al. Identification of clinical outcome measures for recovery of gastrointestinal motility in postoperative ileus. Ann Surg 2014;259:708-14. 
34 Maffezzini M, Campodonico F, Canepa G, et al. Current perioperative management of radical cystectomy with intestinal urinary reconstruction for muscle-invasive bladder cancer and reduction of the incidence of postoperative ileus. Surg Oncol 2008;17:41-8.

35 Bungard TJ, Kale-Pradhan PB. Prokinetic agents for the treatment of postoperative ileus in adults: a review of the literature. Pharmacotherapy 1999;19:416-23.
36 Chae H-D, Kwak M-A, Kim I-H. Effect of acupuncture on reducing duration of postoperative ileus after gastrectomy in patients with gastric cancer: a pilot study using sitz marker. J Altern Complement Med 2016;22:465-72.

37 Vilz TO, Pantelis D, Lingohr P, et al. SmartPill@ as an objective parameter for determination of severity and duration of postoperative ileus: study protocol of a prospective, two-arm, open-label trial (the PIDuSA study). BMJ Open 2016;6:e011014. 